EPJ Web of Conferences 32, 03001 (2012)

DOI: $10.1051 /$ epjconf/20123203001

(C) Owned by the authors, published by EDP Sciences, 2012

\title{
Summary of ECE Presentations at EC-17
}

\author{
M. E. Austin ${ }^{1}$ \\ ${ }^{1}$ Institute for Fusion Studies, University of Texas, Austin, TX 78712, USA
}

\begin{abstract}
At the EC-17 workshop there were 12 presentations in the topic of diagnosing plasmas by measuring radiative emissions, mainly by ECE with one talk on EBW imaging. The number of ECE imaging systems on plasma devices has increased and new discoveries are being made from the expanded data set they provide. Technology continues to improve with some significant advances in receiver capabilities and clever designs for coupling to the plasma. A wide variety of ECE systems on many machines are providing crucial information on electron temperature and other plasma parameters, particularly on fluctuations related to MHD modes, their temporal and spatial structures. The ITER ECE system design is well along with the recent successful completion of the conceptual design review.
\end{abstract}

\section{Imaging measurements}

The number of imaging measurements of plasma waves continues to grow with new systems planned or coming online on several machines. G. Yun presented results of ECE imaging from KSTAR, showing poloidal rotation of modes, dual flux tubes, and structures propagating near the plasma edge. First results from MAST were shown from a microwave imaging system that was looking at EBW emission (R. Vann). The 2-D imaging is accomplished with a novel phased array antenna system that is capable of both passive and active operation. A summary of measurements from the ECE imaging diagnostic on ASDEX-Upgrade was presented (A. Bogomolov) focusing on ELM observations. Rotating filaments associated with the type-I ELM precursor are seen and type-II ELMs show a broadband MHD fluctuation.

\section{EC emission and radiation transport}

A number of papers addressed the basics of electron cyclotron emission and transport. S. Rathgeber presented analysis of radiation temperature from ECE edge emission on ASDEX-Upgrade. A key result is that the bump in $T_{\text {rad }}$ associated with ECE resonances just outside the LCFS in H-mode discharges has been shown to be due to emission from inside the steep gradient region in the plasma. This is extremely important for the ECE imaging diagnostics that are trying to interpret $T_{\mathrm{e}}$ structures associated with the edge. A. White gave a talk on a statistical comparison of Thomson Scattering (TS) and ECE $T_{\mathrm{e}}$ measurements up to $8 \mathrm{keV}$ on C-MOD, looking for the TS/ECE discrepancy seen at high $T_{\mathrm{e}}$ on other tokamaks. No discrepancy was found which motivates a need for modelling to interpret the multi-machine observations.

This is an Open Access article distributed under the terms of the Creative Commons Attribution License 2.0, which permits unrestricted use, distribution, and reproduction in any medium, provided the original work is properly cited. 
A couple of papers addressed non-thermal emission. A new vertical viewing ECE system has been set up on TCV to study the electron distribution function in this tokamak's high power density ECH discharges (W. Eshetu). On the T-10 tokamak, a number of cases of non-thermal emission have been noted with apparent invariant distribution function shape, implying a persistent turbulent state (V. Poznyak). Finally, a paper on the radiative transport of energy via cyclotron resonances was given by F. Albajar, discussing the importance of this effect on ITER for the case of suprathermal electrons.

\section{ECE with heterodyne receivers}

A collection of presentations showed results from new or improved ECE heterodyne radiometer setups. First results from the line-of-sight ECE system incorporated into the ECRH launcher on ASDEX-Upgrade were presented by W. Bongers. This difficult engineering feat is necessary for feedback control of ECRH for NTM suppressing, and the early results are promising. L. Porte gave a talk on correlation ECE (CECE) measurements on TCV. Dramatic reductions in the turbulence correlation length were seen as collisionality was increased and surprising changes in fluctuation spectral content were observed for different z-height views. High frequency MHD modes were studied with the upgraded heterodyne radiometer on Tore Supra and differences in simultaneous half-radius low frequency modes were noted (D. Elbeze). The extensive suite of ECE instruments on T-10 was described by V. Poznyak where simultaneous measurements of 1 st harmonic O-mode and 2nd harmonic X-mode are regularly made. Finally, R. Pavlichenko showed data from a heterodyne receiver measuring optically thin emission from the Uragnan-3M tokamak, demonstrating once again the broad range of usefulness of ECE measurements from magnetized plasmas.

\section{ECE ON ITER}

On the last day of the workshop, the most recent work and progress on the ECE diagnostic system design was presented in the ITER session. An overview of the recently completed conceptual design review was given by $\mathrm{M}$. Austin, showing the design is well along while some issues need to be addressed and decisions need to be made for the transmission line and Michelson interferometer instrument. H. Pandya gave a paper on plans for the back-end instruments and their expected performance. A talk on the application of ECE for control of NTM suppression was given by H. van den Brand, showing that even with relativistic broadening the ECE should have sufficient resolution with appropriate channel layout to assist in the detection of modes. Finally a presentation of the possibilities of extending the capabilities of ECE on ITER by utilizing other millimeter wave access channels such as the reflectometer port, and collective Thomson scattering and ECH launchers (V. Udintsev).

\section{Concluding remarks}

ECE is still an active area for research and development. Two dimensional imaging systems are the putative state-of-the-art diagnostic for magnetically confined plasmas and are providing intriguing new insights. Measurement of coherent and turbulent $T_{\mathrm{e}}$ fluctuations remains the bailiwick of ECE instruments. The ECE/TS discrepancy is still an open question and is in need of modelling work. An ITER-ECE diagnostic design is at hand but several potential problem areas need to be addressed, and in-the-lab tests will be required to verify the solutions. 